\title{
Development of mini core collection of Japanese rice landrace
}

\author{
Kaworu Ebana* $^{* 1)}$, Yoichiro Kojima ${ }^{(1,2)}$, Shuichi Fukuoka ${ }^{1)}$, Tsukasa Nagamine1,3) and Makoto Kawase ${ }^{1)}$ \\ 1) National Institute of Agrobiological Sciences, 2-1-2 Kannondai, Tsukuba, Ibaraki 305-8602, Japan \\ 2) Toyama Agricultural Research Center, 1124-1 Yoshioka, Toyama 939-8153, Japan \\ 3) National Institute of Crop Science, 2-1-18 Kannondai, Tsukuba, Ibaraki 305-8518, Japan
}

\begin{abstract}
A mini core collection of Japanese rice landrace was developed based on a genome-wide SSR polymorphism survey of 236 accessions. The accessions used in the initial survey were selected based on the historical record and passport data from the collection maintained at the Genebank of the National Institute of Agrobiological Sciences (NIAS). These accessions were analyzed using 32 SSR markers. A total of 247 alleles were detected and the number of alleles per locus ranged from 3 to 15 (mean 7.7). Principal coordinate analysis using SSR data enabled classification of the accessions into three major groups, two Japonica groups and one Indica group. To develop a Japanese landrace mini core collection, the SSR data on the 236 accessions were subjected to cluster analysis and 50 groups were recognized at a similarity index of 0.88 . A single accession from each of the 50 groups was selected. These 50 accessions retained $87.5 \%$ of the alleles detected in the original 236 accessions, and covered the variation of the initial set of accessions in terms of several agro-morphological traits.
\end{abstract}

Key Words: Oryza sativa L., Japanese landrace, mini core collection, genetic diversity, SSR.

\section{Introduction}

Crop landraces are valuable genetic resources, because they are considered to contain genetic diversity and therefore should complement and diversify the gene pool of advanced cultivars (Frankel and Soule 1981). Landraces of rice have extensive variation, but only a few have been introduced into elite lines (Yang 1994, Kobayashi et al. 2006, Fukuoka et al. 2006). The enhancement of such germplasm conserved in genebanks for further improvement of rice cultivars is important; however, it is difficult to evaluate many accessions at one time. The idea of a core collection was proposed by Frankel (1984) and is a small set of accessions (usually $10 \%$ of the original size) chosen to represent the genetic spectrum of the whole collection. Recently, a cultivar set smaller than a core collection has been developed as a "mini core collection" or "micro core collection" to evaluate their variation thoroughly (Upadhyaya and Ortiz 2001), because the size of a core collection is sometimes too large for users to handle. The authors developed a small core collection of world rice collection, the Rice Diversity Research Set of NIAS (Kojima et al. 2005). Only two Japanese accessions are included in this set. Japan is located at the edge of rice cultivation, and the genetic diversity of Japanese landraces seems smaller than the world-wide germplasm collection (Kojima et al. 2005); however, the local adapted cultivars

Communicated by K. Okuno

Received March 27, 2008. Accepted June 13, 2008.

*Corresponding author (e-mail: ebana@affrc.go.jp)

$\dagger$ These authors contributed equally to this work are expected to have a different genetic polymorphism to adapt to different environmental conditions. The set of Japanese landraces will be unique and important for understanding rice adaptation in the northern area. One of the objectives of this study was to develop a Japanese landrace mini core collection.

Another problem of using the germplasm is the "redundancy" of accessions. In the Genebank, some accessions with the same variety name have been stored and is difficult to know whether they are redundant or different accessions. Recently, molecular markers have been used for cultivar identification. It is important to identify redundant or different accessions with the same name.

In this study, the genetic variation of Japanese cultivars of landraces was estimated using SSR polymorphism and a representative set of accessions was developed from the Genebank of the National Institute of Agrobiological Sciences (NIAS).

\section{Materials and Methods}

\section{Plant materials}

Among the ca. 2000 Japanese accessions conserved in NIAS Genebank, 236 accessions, including 154 lowland and 82 upland landrace accessions mentioned in the historical records, were selected (Table 1) to cover the whole of Japan. We selected mainly landraces from Genebank accessions, which included 119 accessions with 12 variety names to clarify their "redundancy". Accessions called "Akamai" are not included in the redundancy analysis because of their different morphology and passport data. Eighteen improved 
Table 1. List of accessions

\begin{tabular}{|c|c|c|c|c|c|c|}
\hline JP No. & GB Acc. No. ${ }^{a}$ & Name & landrace/improved & lowland/upland & Diversity Index & Identity $^{b}$ \\
\hline 14908 & & Aichiasahi & improved & lowland & 19.8 & \\
\hline 4982 & & Aikoku & landrace & upland & 20.1 & \\
\hline 5419 & & Aikoku & landrace & lowland & 15.2 & \\
\hline 6365 & & Aikoku & landrace & lowland & 11.6 & 1 \\
\hline 6369 & & Aikoku & landrace & lowland & 12.6 & \\
\hline 6730 & & Aikoku & landrace & lowland & 11.6 & 1 \\
\hline 6768 & & Aikoku & landrace & lowland & 12.7 & \\
\hline 9310 & & Aikoku & landrace & lowland & 17.7 & \\
\hline 9650 & & Aikoku & landrace & lowland & 11.6 & 1 \\
\hline 10993 & & Aikoku & landrace & lowland & 12.2 & \\
\hline 147444 & & Aikoku & landrace & lowland & 11.6 & 1 \\
\hline 5301 & & Akage & landrace & upland & 26.7 & \\
\hline 6729 & & Akage & landrace & lowland & 15.2 & \\
\hline 14994 & & Akage & landrace & upland & 40.3 & \\
\hline 34110 & & Akage & landrace & lowland & 25.4 & \\
\hline 219839 & & Akage & landrace & lowland & 20.9 & \\
\hline 4658 & & Akage & landrace & lowland & 22.3 & \\
\hline 4744 & & Akamai & landrace & upland & 24.2 & \\
\hline 4903 & & Akamai & landrace & upland & 41.8 & \\
\hline 4945 & & Akamai & landrace & upland & 42.4 & \\
\hline 7237 & & Akamai & landrace & lowland & 44.0 & \\
\hline 80563 & & Akamai & landrace & lowland & 42.5 & \\
\hline 9694 & & Akamai & landrace & lowland & 26.3 & \\
\hline 4663 & & Akayakan & landrace & upland & 18.9 & \\
\hline 6183 & & Akitakomachi & improved & lowland & 14.9 & \\
\hline 9707 & & Araki & landrace & lowland & 13.6 & \\
\hline 10736 & & Asahi & landrace & lowland & 14.7 & \\
\hline 14862 & & Asahi & landrace & lowland & 19.9 & \\
\hline 6746 & & Asahi & landrace & lowland & 14.7 & \\
\hline 6761 & & Asahi & landrace & lowland & 17.4 & \\
\hline 6812 & 00007503 & Asahi & landrace & lowland & 13.7 & \\
\hline 6812 & 00007893 & Asahi & landrace & lowland & 13.8 & \\
\hline 6812 & 00007957 & Asahi & landrace & lowland & 13.1 & \\
\hline 6812 & 00016511 & Asahi & landrace & lowland & 14.1 & \\
\hline 9328 & & Asahi & landrace & lowland & 15.4 & \\
\hline 9855 & & Asahi & landrace & lowland & 15.2 & \\
\hline 14996 & & Benkei & selection & lowland & 15.0 & \\
\hline 7199 & & Bouzu & landrace & lowland & 17.3 & \\
\hline 4950 & & Bouzu Mochi & landrace & upland & 16.4 & \\
\hline 6731 & & Bungo & landrace & lowland & 15.3 & \\
\hline 57655 & & Daikoku Wase & improved & lowland & 14.5 & \\
\hline 4860 & & Dango & landrace & upland & 16.9 & \\
\hline 4932 & 00004880 & Dango Mochi & landrace & upland & 18.2 & \\
\hline 4932 & 00005449 & Dango Mochi & landrace & upland & 16.6 & \\
\hline 4932 & 00005655 & Dango Mochi & landrace & upland & 16.8 & \\
\hline 4919 & & Esojima Mochi & landrace & upland & 16.8 & \\
\hline 14924 & & Fukoku & improved & lowland & 18.9 & \\
\hline 7044 & & Fukubouzu & improved & lowland & 17.1 & \\
\hline 4887 & & Fundechangomi & landrace & upland & 17.7 & \\
\hline 14943 & & Gaisenmochi & landrace & upland & 16.8 & \\
\hline 4445 & & Gaisenmochi & landrace & upland & 17.2 & \\
\hline 4705 & & Gaisenmochi & landrace & upland & 21.4 & \\
\hline 4751 & & Gaisenmochi & landrace & upland & 16.3 & \\
\hline 4779 & & Gaisenmochi & landrace & upland & 16.5 & \\
\hline 4914 & & Gaisenmochi & landrace & upland & 17.2 & \\
\hline 5164 & & Gaisenmochi & landrace & upland & 17.7 & \\
\hline 6356 & & Ginbouzu & landrace & lowland & 15.0 & \\
\hline 15142 & & Gohyakumangoku & improved & lowland & 12.7 & \\
\hline
\end{tabular}


Table 1. (continued)

\begin{tabular}{|c|c|c|c|c|c|c|}
\hline JP No. & GB Acc. No. ${ }^{a}$ & Name & landrace/improved & lowland/upland & Diversity Index & Identity $^{b}$ \\
\hline 4819 & & Hakamuri & landrace & upland & 19.4 & \\
\hline 9663 & & Hakutou & landrace & lowland & 20.2 & \\
\hline 4904 & & Hassokuho & landrace & upland & 24.7 & \\
\hline 5769 & & Hattan & landrace & lowland & 16.4 & \\
\hline 6113 & & Himenomochi & improved & lowland & 18.3 & \\
\hline 4916 & & Hinode & landrace & upland & 24.1 & \\
\hline 7446 & & Hinode & landrace & lowland & 12.9 & \\
\hline 54242 & & Hinohikari & improved & lowland & 13.2 & \\
\hline 6363 & & Hinomaru & improved & lowland & 13.9 & \\
\hline 4535 & & Hirayama & landrace & upland & 22.0 & \\
\hline 88635 & & Hitomebore & improved & lowland & 13.3 & \\
\hline 5870 & & Hiyadachitou & landrace & lowland & 18.2 & \\
\hline 11289 & & Hiyokumochi & improved & lowland & 15.5 & \\
\hline 7638 & & HomanJinjaMai & landrace & lowland & 17.1 & \\
\hline 7313 & & Hosogara & landrace & lowland & 15.4 & \\
\hline 4763 & & Irimanishiki & landrace & upland & 22.3 & \\
\hline 7014 & & Ishijiro & landrace & lowland & 17.8 & \\
\hline 4859 & & Isikawa & landrace & upland & 14.8 & \\
\hline 9612 & & Iwai & landrace & lowland & 13.1 & \\
\hline 5906 & & Joushuu & landrace & lowland & 16.9 & \\
\hline 15126 & & Jukkoku & landrace & lowland & 14.4 & \\
\hline 9801 & & Kabashiko & landrace & lowland & 17.0 & \\
\hline 10643 & & Kabashiko & landrace & lowland & 17.8 & \\
\hline 10697 & & Kabashiko & landrace & lowland & 14.2 & \\
\hline 10698 & & Kabashiko & landrace & lowland & 11.9 & \\
\hline 10699 & & Kabashiko & landrace & lowland & 19.9 & \\
\hline 10703 & & Kabashiko & landrace & lowland & 19.6 & \\
\hline 10727 & & Kabashiko & landrace & lowland & 43.8 & \\
\hline 10741 & & Kabashiko & landrace & lowland & 21.2 & \\
\hline 10833 & & Kabashiko & landrace & lowland & 21.6 & \\
\hline 10875 & & Kabashiko & landrace & lowland & 22.7 & \\
\hline 10896 & & Kabashiko & landrace & lowland & 16.3 & \\
\hline 10897 & & Kabashiko & landrace & lowland & 20.3 & \\
\hline 10898 & & Kabashiko & landrace & lowland & 11.6 & \\
\hline 11211 & & Kabashiko & landrace & lowland & 16.5 & \\
\hline 11346 & & Kabashiko & landrace & lowland & 18.2 & \\
\hline 11385 & & Kabashiko & landrace & lowland & 13.0 & \\
\hline 105744 & & Kabashiko & landrace & lowland & 18.8 & \\
\hline 4719 & & Kahei & landrace & upland & 19.1 & \\
\hline 81255 & & Kairyou 41 No.1-1 & landrace & upland & 23.6 & \\
\hline 4798 & 00004940 & Kameji & landrace & upland & 20.6 & \\
\hline 4798 & 00005309 & Kameji & landrace & upland & 16.9 & \\
\hline 7350 & & Kameji & landrace & lowland & 14.7 & \\
\hline 7509 & & Kameji & landrace & lowland & 13.8 & \\
\hline 9329 & & Kameji & landrace & lowland & 12.5 & \\
\hline 9330 & & Kameji & landrace & lowland & 17.1 & \\
\hline 9671 & & Kameji & landrace & lowland & 13.9 & \\
\hline 9886 & & Kameji & landrace & lowland & 20.2 & \\
\hline 9895 & & Kameji & landrace & lowland & 12.9 & \\
\hline 9898 & & Kameji & landrace & lowland & 12.4 & \\
\hline 9905 & & Kameji & landrace & lowland & 12.9 & \\
\hline 14945 & & Kameji & landrace & lowland & 13.8 & \\
\hline 11485 & & Kamenoo & landrace & lowland & 16.5 & \\
\hline 206131 & & Kaneko & landrace & upland & 21.9 & \\
\hline 4403 & & Kaneko & landrace & upland & 20.4 & \\
\hline 4410 & & Kaneko & landrace & upland & 31.7 & \\
\hline 4754 & 00005507 & Kaneko & landrace & upland & 17.6 & \\
\hline 4754 & 00005508 & Kaneko & landrace & upland & 16.9 & \\
\hline
\end{tabular}


Table 1. (continued)

\begin{tabular}{|c|c|c|c|c|c|c|}
\hline JP No. & GB Acc. No. ${ }^{a}$ & Name & landrace/improved & lowland/upland & Diversity Index & Identity $^{b}$ \\
\hline 4817 & 00004937 & Kaneko & landrace & upland & 26.0 & \\
\hline 4817 & 00005329 & Kaneko & landrace & upland & 22.0 & \\
\hline 4929 & & Kaneko & landrace & upland & 17.5 & \\
\hline 4979 & & Kaneko & landrace & upland & 20.2 & \\
\hline 10788 & & Karahoushi & landrace & lowland & 46.8 & \\
\hline 4897 & & Kasanari Bouzu & landrace & upland & 19.6 & \\
\hline 4726 & & Ke Mochi & landrace & upland & 18.5 & \\
\hline 9622 & & Kinai Omachi 1 & landrace & lowland & 14.3 & \\
\hline 9626 & & Kinai Omachi 2 & landrace & lowland & 17.0 & \\
\hline 4748 & & Kirishima & landrace & upland & 19.3 & \\
\hline 7449 & & Kiryouyoshi & landrace & lowland & 14.7 & \\
\hline 6300 & & Koganemochi & improved & lowland & 14.3 & \\
\hline 7286 & & Kokuryoumiyako & landrace & lowland & 15.5 & \\
\hline 6460 & & Koshihikari & improved & lowland & 13.1 & \\
\hline 4791 & & Kurohige & landrace & upland & 15.6 & \\
\hline 4895 & & Kuroka & landrace & upland & 22.2 & \\
\hline 4482 & & Kurumi Wase & landrace & upland & 21.8 & \\
\hline 6735 & & Mansaku & landrace & lowland & 17.8 & \\
\hline 7405 & & Masaoka & landrace & lowland & 11.1 & \\
\hline 4923 & & Meguro Mochi & landrace & upland & 18.1 & \\
\hline 7448 & & Mikuni & landrace & lowland & 13.9 & \\
\hline 6876 & & Mitsui & improved & lowland & 14.2 & \\
\hline 7450 & & Miyako & landrace & lowland & 13.1 & \\
\hline 147541 & & Miyamanishiki & improved & lowland & 11.7 & \\
\hline 4828 & & Miyanishiki & landrace & upland & 17.6 & \\
\hline 7318 & & Moritawase & landrace & lowland & 16.5 & \\
\hline 14931 & & Mubouaikoku & landrace & lowland & 11.4 & \\
\hline 4653 & & Nagae Wase & landrace & upland & 21.4 & \\
\hline 7227 & & Nagoyashiro & landrace & lowland & 17.8 & \\
\hline 222430 & & Nipponbare & improved & lowland & 13.1 & \\
\hline 4659 & & Oiran & landrace & upland & 22.2 & \\
\hline 4951 & & Okabo & landrace & upland & 16.8 & \\
\hline 4783 & & Okatama Nishiki & landrace & upland & 38.6 & \\
\hline 4805 & & Okka Modoshi & landrace & upland & 21.2 & \\
\hline 4551 & & Omochi & landrace & upland & 20.0 & \\
\hline 7529 & & Omochi & landrace & lowland & 13.8 & \\
\hline 9872 & & Omochi & landrace & lowland & 14.4 & \\
\hline 14946 & & Omochi & landrace & lowland & 17.1 & \\
\hline 6319 & & Oobamochi & landrace & lowland & 15.1 & \\
\hline 4781 & & Oohata Wase & landrace & upland & 20.2 & \\
\hline 6906 & & Ouba & landrace & lowland & 17.6 & \\
\hline 4731 & & Owari Mochi & landrace & upland & 17.8 & \\
\hline 4833 & & Raiden & landrace & upland & 18.0 & \\
\hline 69789 & & Rikutou Rikuu 2 & landrace & upland & 17.8 & \\
\hline 5416 & & Rikuu 132 & improved & lowland & 14.4 & \\
\hline 7117 & & Sakai Kaneko & landrace & lowland & 15.0 & \\
\hline 15034 & & Sekitori & landrace & lowland & 11.7 & \\
\hline 4604 & 00005019 & Sekiyama & landrace & upland & 27.0 & \\
\hline 4604 & 00005091 & Sekiyama & landrace & upland & 27.0 & \\
\hline 5447 & & Sekiyama & landrace & lowland & 22.2 & \\
\hline 5448 & & Sekiyama & landrace & lowland & 16.5 & \\
\hline 5541 & & Sekiyama & landrace & lowland & 22.2 & \\
\hline 5542 & & Sekiyama & landrace & lowland & 23.2 & \\
\hline 5543 & 00006131 & Sekiyama & landrace & lowland & 16.8 & \\
\hline 5543 & 00006133 & Sekiyama & landrace & lowland & 16.7 & \\
\hline 5544 & & Sekiyama & landrace & lowland & 17.4 & \\
\hline 5546 & & Sekiyama & landrace & lowland & 16.1 & \\
\hline 5753 & & Sekiyama & landrace & lowland & 16.6 & \\
\hline
\end{tabular}


Table 1. (continued)

\begin{tabular}{|c|c|c|c|c|c|c|}
\hline JP No. & GB Acc. No. ${ }^{a}$ & Name & landrace/improved & lowland/upland & Diversity Index & Identity $^{b}$ \\
\hline 7041 & & Sekiyama & landrace & lowland & 17.0 & \\
\hline 7285 & & Sekiyama & landrace & lowland & 16.5 & \\
\hline 7537 & & Sekiyama & landrace & lowland & 12.4 & \\
\hline 11540 & & Sekiyama & landrace & lowland & 17.8 & \\
\hline 4674 & & Senbon & landrace & upland & 21.7 & \\
\hline 4418 & & Senbonmochi & landrace & upland & 22.2 & \\
\hline 7278 & & Senichi & landrace & lowland & 15.6 & \\
\hline 14334 & & Sensyou & landrace & upland & 18.2 & 2 \\
\hline 4385 & & Sensyou & landrace & upland & 17.5 & \\
\hline 4386 & & Sensyou & landrace & upland & 18.6 & 3 \\
\hline 4522 & & Sensyou & landrace & upland & 17.9 & 4 \\
\hline 4704 & & Sensyou & landrace & upland & 17.9 & 4 \\
\hline 4893 & & Sensyou & landrace & upland & 18.6 & 3 \\
\hline 4948 & & Sensyou & landrace & upland & 18.2 & 2 \\
\hline 5108 & & Sensyou & landrace & upland & 18.4 & \\
\hline 4958 & & Seridashi & landrace & upland & 18.2 & \\
\hline 4938 & & Shichimenchou Mochi & landrace & upland & 23.7 & \\
\hline 7494 & & Shikishima & landrace & lowland & 11.8 & \\
\hline 4747 & & Shindai Okoshi & landrace & upland & 38.0 & \\
\hline 4464 & 00004879 & Shinriki & landrace & upland & 23.1 & \\
\hline 4464 & 00005729 & Shinriki & landrace & upland & 19.5 & \\
\hline 6758 & & Shinriki & landrace & lowland & 12.6 & \\
\hline 7221 & & Shinriki & landrace & lowland & 15.0 & \\
\hline 7539 & & Shinriki & landrace & lowland & 14.0 & \\
\hline 9109 & & Shinriki & landrace & lowland & 12.4 & \\
\hline 9411 & & Shinriki & landrace & lowland & 14.5 & \\
\hline 10754 & & Shinriki & landrace & lowland & 13.6 & \\
\hline 11005 & & Shinriki & landrace & lowland & 12.6 & \\
\hline 9623 & & Shinyamadaho 1 & landrace & lowland & 13.1 & \\
\hline 6962 & & Shinyamadaho 2 & landrace & lowland & 19.0 & \\
\hline 7602 & & Shiratamamochi & landrace & lowland & 16.6 & \\
\hline 7104 & & Shirodama & landrace & lowland & 11.4 & \\
\hline 7280 & & Shirozasa & landrace & lowland & 13.4 & \\
\hline 4888 & & Shrohige & landrace & upland & 19.5 & \\
\hline 7334 & & Sinrikimochi & landrace & lowland & 13.1 & \\
\hline 7523 & & Sinrikimochi & landrace & lowland & 16.9 & \\
\hline 9416 & & Sinrikimochi & landrace & lowland & 15.6 & \\
\hline 9465 & & Sinrikimochi & landrace & lowland & 17.9 & \\
\hline 10891 & & Sinrikimochi & landrace & lowland & 16.9 & \\
\hline 10908 & & Sinrikimochi & landrace & lowland & 17.5 & \\
\hline 14937 & & Sinrikimochi & landrace & lowland & 14.8 & \\
\hline 34021 & & Sinrikimochi & landrace & lowland & 15.5 & \\
\hline 42863 & & Sinrikimochi & landrace & lowland & 15.8 & \\
\hline 7369 & & Sinsyuu & landrace & lowland & 18.5 & \\
\hline 7497 & & Sinsyuukaneko & landrace & lowland & 16.9 & \\
\hline 7293 & & Soutoku & landrace & lowland & 17.9 & \\
\hline 7297 & & Sugaippon & landrace & lowland & 17.5 & \\
\hline 14947 & & Takedawase & landrace & lowland & 11.9 & \\
\hline 7279 & & Takenari & landrace & lowland & 14.3 & \\
\hline 4869 & & Tamasari & landrace & upland & 14.9 & \\
\hline 10772 & & Touboshi & landrace & lowland & 47.8 & \\
\hline 6038 & & Tougou & landrace & lowland & 15.6 & \\
\hline 4700 & & Touzou Mochi & landrace & upland & 20.4 & \\
\hline 6912 & & Toyokuni & landrace & lowland & 19.2 & \\
\hline 4826 & & Urasan & landrace & upland & 17.4 & \\
\hline 100383 & & Wataribune & landrace & lowland & 23.7 & \\
\hline 6957 & & Yajouho & landrace & lowland & 13.1 & \\
\hline 4839 & & Yamada Bake & landrace & upland & 23.3 & \\
\hline
\end{tabular}


Table 1. (continued)

\begin{tabular}{|c|c|c|c|c|c|c|}
\hline JP No. & GB Acc. No. ${ }^{a}$ & Name & landrace/improved & lowland/upland & Diversity Index & Identity $^{b}$ \\
\hline 100487 & & Yamadaho & landrace & lowland & 13.9 & \\
\hline 6958 & & Yamadanishiki & improved & lowland & 13.2 & \\
\hline 7291 & & Yamatonishiki & landrace & lowland & 17.2 & \\
\hline 4666 & & Yorunoyuki & landrace & upland & 17.6 & \\
\hline
\end{tabular}

${ }^{a} \mathrm{~GB}$ accession No. is added when JP No. is same as other accession.

${ }^{b}$ Same number shows that their SSR profile is same and Diversity index is equal.

varieties were used as a control. Modern varieties from crosses were designated as 'improved variety', and varieties selected from landraces were included in 'landrace'. Information on cultivation type, upland or lowland, followed the passport data of Genebank of NIAS.

\section{DNA extraction and SSR analysis}

Total DNA was extracted from young leaves of 10 plants in each accession with the CTAB method (Murray and Thompson 1980). When analyzing the genetic diversity of accessions, DNA is amplified in a final volume of $10 \mu \mathrm{l}$ containing $10 \mathrm{mM}$ Tris- $\mathrm{HCl}(\mathrm{pH} 8.3)$ and $50 \mathrm{mM} \mathrm{KCl}$ (Perkin Elmer), $2.5 \mathrm{mM} \mathrm{MgCl}_{2}$ and $2 \mathrm{mM}$ dNTPs, $4 \mu \mathrm{M}$ primer pairs, $5 \mathrm{ng}$ total DNA and 0.5 unit of AmpliTaq Gold DNA Polymerase (Applied Biosystems). The amplification program was as follows: initial incubation at $95^{\circ} \mathrm{C}$ for $9 \mathrm{~min}$, followed by 35 cycles of denaturation at $94^{\circ} \mathrm{C}$ for $30 \mathrm{sec}$, annealing at $55^{\circ} \mathrm{C}$ for $1 \mathrm{~min}$, extension at $72^{\circ} \mathrm{C}$ for $2 \mathrm{~min}$, and final extension at $72^{\circ} \mathrm{C}$ for $10 \mathrm{~min}$. PCR products $(1.5 \mu \mathrm{l})$ were denatured at $95^{\circ} \mathrm{C}$ for 2 minutes, and electrophoresed on $4.5 \%$ polyacrylamide gel containing $7 \mathrm{M}$ urea in $1 \times \mathrm{TBE}$ buffer at 2,000 V for $80 \mathrm{~min}$. Gels were stained with Vistra Green (Amersham Pharmacia Biotech) and detected by image scanner machine STORM 860 (Amersham Pharmacia Biotech). From the previous report on SSR analysis of rice (Akagi et al. 1997, Chen et al. 1997, Tenmykh et al. 2000, Tenmykh et al. 2001), 32 pairs of primers were selected for analysis (Table 2) to cover the genome with a small number of markers which could detect polymorphism in Japonica accessions.

\section{Evaluation of genetic diversity}

To estimate genetic difference among accessions, the diversity index of each accession was calculated as follows.

Diversity index $=-\log _{10} \prod_{i=1}^{N} p i$, where $p i$ is the allele frequency of each SSR allele at locus $i$ in the total accessions. This index shows the standard and outlier combination of alleles in the samples. This index was also calculated for each same variety name group to estimate "redundancy".

\section{Selection of mini core collection}

Each of the SSR alleles was scored for presence (1) or absence (0). A similarity index was calculated using a simple matching coefficient of similarity (Sokal and Michener 1958 ) with these 1-0 data. Principal coordinate analysis using the coefficient was performed with NTSYS ver. 2.02
(Exeter Software). A dendrogram was generated from a pairwise distance matrix based on the unweighted pair-group method by arithmetic means (UPGMA) (Sneath and Sokal 1973) to reveal genetic relationships among accessions, using the software SPSS version 11.5 (SPSS Inc., USA). A total of 236 accessions, including "redundant" accessions, were used as a representative population. The number of accessions to be included in the collection was decided to retain nearly $90 \%$ of the alleles detected in 236 accessions. After constructing a dendrogram of 236 accessions, the accessions were divided according to the branching pattern of the dendrogram at a given coefficient level. One representative with highest diversity index at a branch was selected to retain as many alleles as possible. To decide the number of mini core collections, the percentage of alleles covered by the selected samples was counted and compared with the random sampling simulation. In the simulation, twenty trials were performed with each number of samples.

In order to evaluate the level of phenotypic diversity in the mini core collection, variation in the phenotype for 9 traits, including culm length, panicle length, number of panicles per plant, grain length, grain width, brown rice length, brown rice width, days to heading from seeding, and amylose content, was evaluated. These characters were evaluated in 2003, in Tsukuba, Ibaraki, Japan (N36 $03^{\circ}$, E140 $\left.10^{\prime}\right)$ under natural field conditions. One-month-old seedlings were transplanted to the paddy field in mid-May and ten plants were used for the evaluation. The mean, standard deviation, and the range of traits in the intensive collection and the original 236 accessions were compared.

\section{Results}

In the present study, 236 rice varieties were analyzed using 32 SSR markers. The number of alleles investigated ranged from 3 to 15 (mean 7.7) per locus and the total number of alleles detected was 247 (Table 2). The most variable locus was RM539 on chromosome 6 with 15 alleles and the next was RM259 on chromosome 1 with 14 allele. Most of the accessions showed different SSR profiles. Two accessions of Kameji, four accessions of Aikoku, and two accessions of Senshou showed the same SSR profiles (Table 1). The diversity index of each accession in all samples was from 11.1 to 47.8 (mean 18.2). Toboshi showed the highest value and "Akamai" accessions showed a rather high value. Masaoka showed the lowest value and "Aikoku" accessions 
generally showed a low value (Table 1).

\section{Redundant accessions}

SSR profiles of 119 accessions of 12 variety name groups were compared (Table 3 ). In the variety name groups of Sekiyama, Shinriki, Kabashiko and Akage, more than $90 \%$ of loci were polymorphic. Sekiyama accessions showed the highest variation (31/32 loci were polymorphic).

Table 2. Number of alleles detected at each SSR marker loci.

\begin{tabular}{|c|c|c|c|c|c|c|}
\hline Marker Name & Repeat Motif & Chr. & Position $(\mathrm{Mb})$ & Product Size & Number of alleles & Source \\
\hline$R M 220$ & (CT)17 & 1 & 4.4 & $100-130$ & 11 & $\mathrm{a}$ \\
\hline$R M 259$ & (CT) 17 & 1 & 7.4 & $148-176$ & 14 & $\mathrm{~b}$ \\
\hline$R M 237$ & (CT) 18 & 1 & 29.2 & $124-138$ & 7 & $\mathrm{~b}$ \\
\hline$R M 84$ & (TCT) 10 & 1 & 31.5 & $118-124$ & 4 & $\mathrm{~b}$ \\
\hline$R M 236$ & (CT)18 & 2 & 2.1 & $190-196$ & 4 & $\mathrm{~b}$ \\
\hline RM208 & (CT) 17 & 2 & 35.2 & $164-176$ & 4 & $\mathrm{a}$ \\
\hline RM207 & (CT) 25 & 2 & 35.4 & $110-132$ & 10 & $\mathrm{~b}$ \\
\hline$R M 213$ & $(\mathrm{CT}) 17$ & 2 & 35.5 & $126-150$ & 6 & a \\
\hline RM571 & (GT)11(AG)13 & 3 & 34.0 & $182-192$ & 4 & $\mathrm{c}$ \\
\hline RM518 & (TC) 15 & 4 & 2.0 & $158-180$ & 9 & $\mathrm{c}$ \\
\hline$R M 241$ & $(\mathrm{CT}) 31$ & 4 & 27.4 & $124-142$ & 10 & $\mathrm{a}$ \\
\hline$R M 255$ & $(\mathrm{AGG}) 5(\mathrm{AG}) 2-(\mathrm{GA}) 16$ & 4 & 31.0 & $130-154$ & 6 & $\mathrm{~b}$ \\
\hline$R M 440$ & (CTT) 22 & 5 & 19.8 & $161-217$ & 13 & $\mathrm{c}$ \\
\hline$R M 480$ & (AC) 30 & 5 & 27.5 & $199-221$ & 12 & $\mathrm{c}$ \\
\hline$R M 217$ & (CT) 20 & 6 & 4.2 & $114-144$ & 11 & $\mathrm{~b}$ \\
\hline RM539 & (TAT) 21 & 6 & 8.2 & $249-289$ & 15 & $\mathrm{c}$ \\
\hline$R M 412$ & $(\mathrm{GA}) 22$ & 6 & 30.3 & $176-200$ & 11 & $\mathrm{c}$ \\
\hline$R M 418$ & (ATT) 21 & 7 & 18.8 & $245-290$ & 11 & $\mathrm{c}$ \\
\hline RM560 & (CT) 12 & 7 & 20.2 & $224-240$ & 8 & $\mathrm{c}$ \\
\hline$R M 25$ & (GA) 18 & 8 & 4.4 & $120-124$ & 3 & $\mathrm{~b}$ \\
\hline$R M 25$ & $(\mathrm{GA}) 18$ & 8 & 4.4 & $146-164$ & 6 & $\mathrm{~b}$ \\
\hline RM515 & $(\mathrm{GA}) 11$ & 8 & 20.4 & $211-219$ & 5 & $\mathrm{c}$ \\
\hline$R M 219$ & (CT) 17 & 9 & 8.5 & $184-204$ & 9 & $\mathrm{~b}$ \\
\hline$R M 201$ & (CT) 17 & 9 & 20.8 & $136-150$ & 4 & $\mathrm{~b}$ \\
\hline$R M 215$ & (CT) 16 & 9 & 22.0 & $146-160$ & 7 & $\mathrm{~b}$ \\
\hline$R M 216$ & (CT) 18 & 10 & 5.0 & $132-154$ & 5 & $\mathrm{~b}$ \\
\hline$R M 228$ & (CA)6(GA)36 & 10 & 21.8 & $100-122$ & 8 & $\mathrm{~b}$ \\
\hline$R M 20 B$ & (ATT) 14 & 11 & 0.9 & $98-116$ & 8 & $\mathrm{~b}$ \\
\hline RM536 & (CT) 16 & 11 & 8.9 & $220-230$ & 6 & $\mathrm{c}$ \\
\hline RM202 & $(\mathrm{CT}) 30$ & 11 & 8.9 & $166-186$ & 7 & $\mathrm{~b}$ \\
\hline$R M 224$ & $(\mathrm{AAG}) 8(\mathrm{AG}) 13$ & 11 & 29.5 & $124-142$ & 6 & $\mathrm{~b}$ \\
\hline$R M 270$ & $(\mathrm{GA}) 13$ & 12 & 25.2 & $210-224$ & 3 & $\mathrm{a}$ \\
\hline
\end{tabular}

a Temnykh et al. 2000, b Chen et al. 1997, c Temnykh et al. 2001.

Table 3. Genetic variation among same named accessions

\begin{tabular}{lccccc}
\hline \hline Classification & $\begin{array}{c}\text { Number of } \\
\text { accession }\end{array}$ & $\begin{array}{c}\text { Polymorphic } \\
\text { loci }(\%)\end{array}$ & $\begin{array}{c}\text { Number of allele per } \\
\text { locus }\end{array}$ & $\begin{array}{c}\text { Number of allele per } \\
\text { locus per accession }\end{array}$ & $\begin{array}{c}\text { Diversity Index } \\
\text { AVE }( \pm \text { SD })\end{array}$ \\
\hline Aikoku & 10 & 78 & 2.1 & 0.21 & $5.5 \pm 5.4$ \\
Akage & 6 & 91 & 2.4 & 0.40 & $9.4 \pm 3.2$ \\
Asahi ${ }^{a}$ & 10 & 84 & 2.0 & 0.20 & $5.7 \pm 2.7$ \\
Gaisen-mochi & 10 & 41 & 1.5 & 0.15 & $3.2 \pm 2.1$ \\
Kabashiko & 17 & 91 & 3.0 & 0.18 & $11.4 \pm 1.5$ \\
Kameji & 12 & 88 & 2.5 & 0.21 & $8.8 \pm 4.1$ \\
Kaneko ${ }^{b}$ & 9 & 71 & 2.3 & 0.25 & $7.5 \pm 4.6$ \\
Omachi & 4 & 97 & 1.8 & 0.45 & $6.4 \pm 4.7$ \\
Sekiyama & 15 & 16 & 1.2 & 0.21 & $11.6 \pm 5.0$ \\
Sensyou & 8 & 94 & 2.3 & 0.14 & $1.2 \pm 0.4$ \\
Shinriki & 9 & 81 & 2.3 & 0.25 & $9.0 \pm 4.2$ \\
Shinriki-mochi & 9 & & & 0.26 & $9.0 \pm 0.7$ \\
\hline
\end{tabular}

${ }^{a}$ Asahi includes "Kyoto Asahi".

${ }^{b}$ Kaneko includes "Tokyo Kaneko". 
On the other hand, Senshou and Gaisenmochi showed only 16 and $41 \%$ polymorphic loci. Among accessions with the same variety name, Sekiyama, Shinriki, Kameji and Aikoku, upland accessions and lowland accessions showed a different diversity index, suggesting two types in each variety group (Fig. 1).

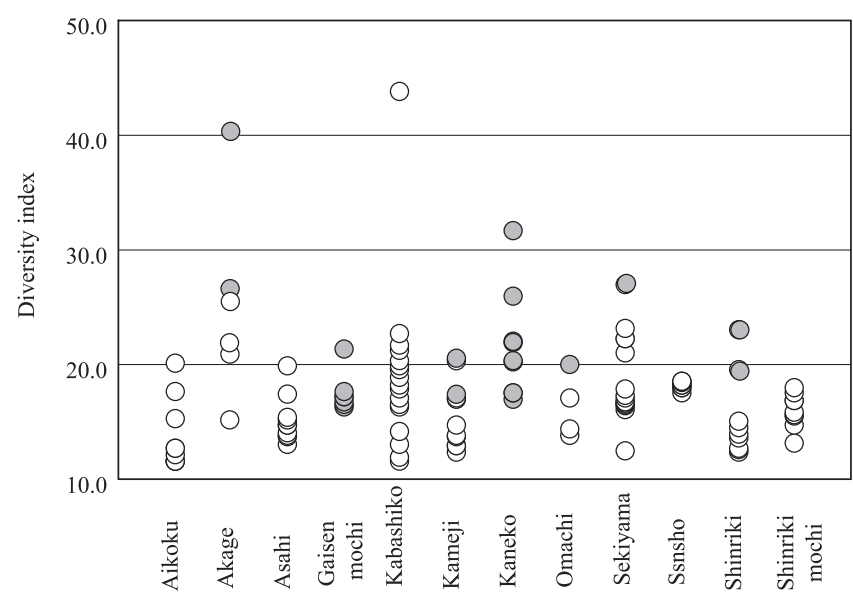

Fig. 1. Intragroup variation in the same name cultivar group. $\bigcirc$ lowland, $\bigcirc$ upland accessions.
Genetic differentiation among Japanese rice landrace

PCA analysis of the 236 accessions showed two different types of variety groups in Japanese landraces, Japonica and Indica. The Japonica group was divided into two groups, Group I and II (Fig. 2). This grouping well matched the cultivation method, lowland and upland. Group I was a mixture of upland and lowland rice varieties and group II consisted only of upland rice. The major varieties in Japan are included in Group I. Markers which could signify Indica/Japonica were RM 207 and RM 208 on chr. 2, RM 217 on chr. 6 and $R M 202$ on chr.11. Markers which could signify lowland/ upland were $R M 236, R M 207$ on chr.2, RM 440 on chr. 5, $R M 412$ on chr.6, RM 418 on chr.7, RM216 on chr.10, RM 536 and $R M 20 B$ on chr.11. Improved varieties showed less genetic diversity (Table 4) and all of the alleles detected in the improved varieties were also detected in landraces (data not shown).

\section{Selection of mini core collection}

In a comparison of statistic and random sampling, the mini core collection of 50 samples was superior to cover the genetic diversity of Japanese landraces (Fig. 3). Fifty Japanese rice accessions, including 216 alleles of these 32 loci,

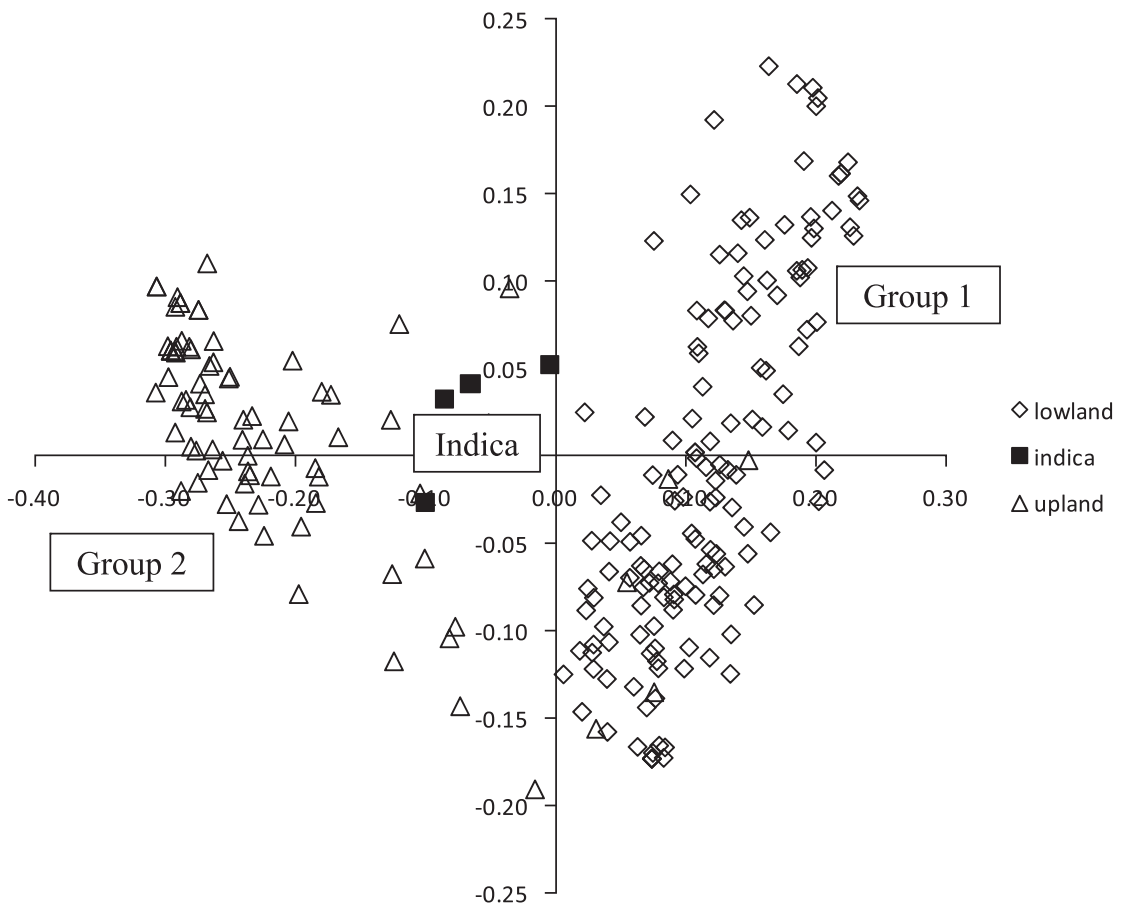

Fig. 2. Three groups of Japanese landrace accessions.

Table 4. Comparison of genetic diversity between improved varieties and landraces

\begin{tabular}{lcccc}
\hline \hline Classification & Number of accession & Polymorphic loci (\%) & Total number of alleles & Diversity Index \\
\hline Improved variety $^{a}$ & 18 & 87.5 & 60 & 14.8 \\
(Leading variety) $^{b}$ & $(4)$ & $(31.3)$ & $(42)$ & $(13.5)$ \\
Landrace & 218 & 100 & 247 & 18.4 \\
\hline
\end{tabular}

a "Improved variety" was defined as the result of crossbreeding.

$b$ "Leading variety" includes Koshihikati, Hitomebore, Akitakomachi and Hinohikari. 


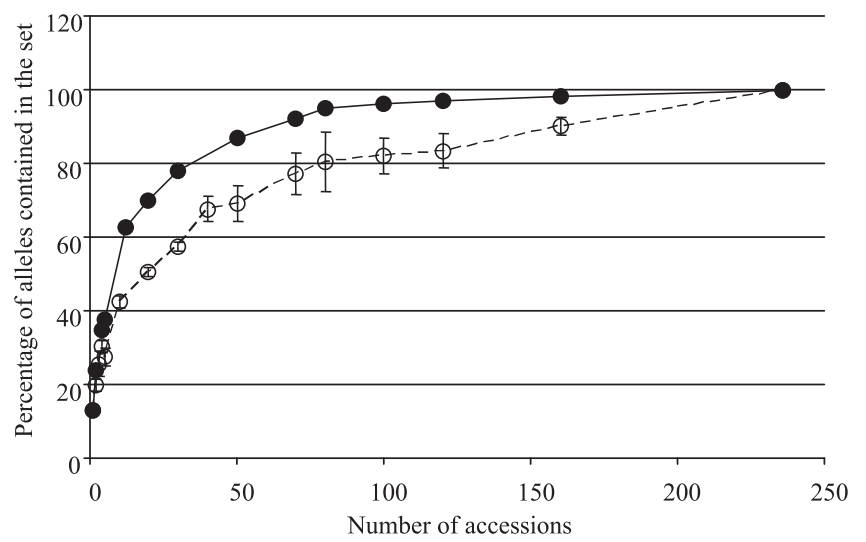

Fig. 3. Percentage of alleles contained in the accession set according to the sample size. Statistic sampling, $\bigcirc$ random sampling.

$87.5 \%$ of the population, were selected as a representative set (Table 5). Comparison of the phenotypic variation in several agro-morphological traits between the collection and the original accessions showed that this collection included 75$100 \%$ of the range of variation (Table 6).

\section{Discussion}

The objective of the present study was to investigate the genetic diversity of Japanese landrace accessions and to develop a mini core collection. Fifty accessions were selected for the collection (Table 5), based on SSR data, and included $87.5 \%$ of the SSR alleles in the 236 accessions.

Among the 32 SSR loci investigated here, some loci showed higher levels of allelic diversity. The presence of highly diverse loci is attributed to the linkage with balanced selective genes (Ishimaru et al. 2001) or an allelic barrier (Harushima et al. 2002). The most variable locus was RM539 on chromosome 6 with 15 alleles located at $8.2 \mathrm{Mb}$ of chromosome 6. $\mathrm{Hdl}$, one of the major genes controlling the heading date is located in this chromosomal region. Japan is at the northern edge of rice cultivation and heading date seems a selective trait in Japan. The next polymorphic marker was RM259 on chromosome 1 with 14 alleles and $R f-3$, which is a restorer gene of CMS located in this region (Zhang et al. 1997). RFLP analysis of the world rice collection did not detect a relationship among polymorphic markers and genes (Kojima et al. 2005). This suggests that the association of markers and genes should be analyzed in genetically similar populations.

As for the Indica/Japonica marker, $H d 7$, which controls the heading date, is located near RM207 and RM208 on chr2. $H d 3 a, R F T 1$ and $w x$ are located near RM217. The heading date and waxiness of the grain (Olsen et al. 2006) are thought to be artificially selective traits. As QTLs in upland/ lowland marker regions, QTL for phosphorous sensitivity (Wissuwa et al. 1998) near RM207, field resistance for rice blast near RM418 (Tabien et al. 2002), spikelet number, amylose content (Cui et al. 2002) and allelopathy (Ebana et
Table 5. List of Japanese landrace mini core collection

\begin{tabular}{|c|c|c|}
\hline ID & Variety Name & upland/lowland \\
\hline JRC 01 & Gaisen Mochi & upland \\
\hline JRC 03 & Hinode & upland \\
\hline JRC 04 & Senshou & upland \\
\hline JRC 05 & Yamada Bake & upland \\
\hline JRC 06 & Kaneko & upland \\
\hline JRC 07 & Irima Nishiki & upland \\
\hline JRC 08 & Okka Modoshi & upland \\
\hline JRC 10 & Hirayama & upland \\
\hline JRC 11 & Kahei & upland \\
\hline JRC 12 & Oiran & upland \\
\hline JRC 13 & Bouzu Mochi & upland \\
\hline JRC 14 & Meguro Mochi & upland \\
\hline JRC 17 & Akage & lowland \\
\hline JRC 18 & Hassokuho & upland \\
\hline JRC 19 & Wataribune & lowland \\
\hline JRC 20 & Hosogara & lowland \\
\hline JRC 21 & Akamai & lowland \\
\hline JRC 22 & Mansaku & lowland \\
\hline JRC 23 & Ishijiro & lowland \\
\hline JRC 24 & Joushuu & lowland \\
\hline JRC 25 & Dango & upland \\
\hline JRC 26 & Aikoku & lowland \\
\hline JRC 27 & Ginbouzu & lowland \\
\hline JRC 28 & Shinriki Mochi & lowland \\
\hline JRC 29 & Shichimenchou Mochi & upland \\
\hline JRC 30 & Morita Wase & lowland \\
\hline JRC 31 & Kameji & lowland \\
\hline JRC 32 & Omachi & lowland \\
\hline JRC 33 & Shinriki & lowland \\
\hline JRC 34 & Kyoutoasahi & lowland \\
\hline JRC 35 & Kabashiko & lowland \\
\hline JRC 36 & Sekiyama & lowland \\
\hline JRC 37 & Shinyamadaho 2 & lowland \\
\hline JRC 38 & Nagoya Shiro & lowland \\
\hline JRC 39 & Shiroine (Kemomi) & upland \\
\hline JRC 40 & Akamai & upland \\
\hline JRC 41 & Akamai & lowland \\
\hline JRC 42 & Touboshi & lowland \\
\hline JRC 43 & Akamai & upland \\
\hline JRC 44 & Karahoushi & lowland \\
\hline JRC 45 & Hiyadachitou & lowland \\
\hline JRC 46 & Fukoku & lowland \\
\hline JRC 47 & Okabo & upland \\
\hline JRC 48 & Hakamuri (Yokoyama) & upland \\
\hline JRC 49 & Rikutou Rikuu 2 & upland \\
\hline JRC 50 & Himenomochi & lowland \\
\hline JRC 51 & Shinshuu & lowland \\
\hline JRC 52 & Aichiasahi & lowland \\
\hline JRC 53 & Raiden & upland \\
\hline JRC 54 & Houmanshinden Ine & lowland \\
\hline
\end{tabular}

al. 2001) near RM412, UV-B resistance (Sato et al. 2003) near RM516, culm strength under upland conditions (Mu et al. 2004) near $R M 440$, were reported. These QTLs seem related to the upland and lowland cultivation conditions or differences among the morphological differences of upland and lowland varieties. This result suggested an association 
Table 6. Comparison of means and ranges for morphological descriptors between the core collection and the initial accessions

\begin{tabular}{lccc}
\hline \hline Descriptors & Mean $\pm \mathrm{SE}^{a}$ & Range & $\begin{array}{c}\text { Retention }^{b} \\
(\%)\end{array}$ \\
\hline Culm length & $102 \pm 13$ & $68-143$ & 100 \\
& $68 \pm 17$ & $68-143$ & \\
\hline Panicle length & $21.3 \pm 1.9$ & $16.6-28.7$ & 85 \\
& $22.2 \pm 2.2$ & $18.4-28.7$ & \\
\hline Number of panicles & $10.7 \pm 2.7$ & $4.8-19.6$ & 99 \\
$\quad$ per plant & $11.5 \pm 3.4$ & $5.0-19.6$ & \\
\hline Grain length & $6.0 \pm 0.4$ & $5.2-7.0$ & 92 \\
& $6.0 \pm 0.4$ & $5.3-7.0$ & \\
\hline Grain width & $3.4 \pm 0.2$ & $2.8-3.8$ & 95 \\
& $3.4 \pm 0.2$ & $2.8-3.8$ & \\
\hline Brown rice length & $5.3 \pm 0.4$ & $4.6-6.4$ & 97 \\
\hline Brown rice width & $5.3 \pm 0.4$ & $4.6-6.4$ & \\
& $2.9 \pm 0.2$ & $2.4-3.4$ & 75 \\
\hline Heading from sawing & $116 \pm 12$ & $80-135$ & 100 \\
\hline Amylose content & $115 \pm 14$ & $80-135$ & \\
\hline
\end{tabular}

${ }^{a}$ Upper lines and lower lines represent the initial collection and the core collection, respectively.

${ }^{b}$ Retention shows the percentage of the range preserved in the core collection.

among SSR markers and selective genes in Japanese rice landraces.

Comparison among accessions with the same variety name shows "redundant" accessions but they generally showed different SSR profiles (Table 1). In the two types of cultivation varieties included in the group, lowland and upland cultivated accessions were critically different (Fig. 1). The present study showed that upland and lowland types are genetically different even if they are have the same variety name and should be considered not as redundant accessions but as different accessions. This result supports the importance of passport data and also the usefulness of molecular analysis of genetic diversity.

Genetic differentiation of Japanese rice landraces was estimated from the present result. Accessions were divided into three groups, two Japonica groups (Group I and II) and one Indica (Fig. 2). Group I includes both upland and lowland accessions, whereas Group II includes only upland accessions. Upland varieties in the Group II showed morphological characters such as thick culms, a small number of panicles and broad leaves, which suggested that they are tropical Japonica. The previous study of world-wide accessions with RFLP analysis did not clearly divide temperate and tropical Japonica (Kojima et al. 2005). SSR can divide these two variety groups and they are a better tool to identify relative varieties than RFLP, because SSRs change rapidly (Charlsworth et al. 1994).

We selected a mini core collection of Japanese rice landraces (Table 5). This set includes 21 upland and 29 lowland accessions and covers $87.5 \%$ of the alleles. It also includes more than $90 \%$ of phenotypic variation in the original population except brown rice width (Table 6). This mini core collection of Japanese landraces is distributed by NIAS Genebank (core collections in NIAS Genebank: http:// www.gene.affrc.go.jp/plant/core_collections.html; contact address: genebank@nias.affrc.go.jp). The SSR genotype and phenotype data are shown on the website. Accumulated data on the various traits and DNA polymorphism in the collection will enhance the efficient use of rice genetic resources.

\section{Acknowledgements}

The authors are grateful to the Genebank of the National Institute of Agrobiological Sciences for providing the germplasm. This work was supported by a grant from the Ministry of Agriculture, Forestry and Fisheries of Japan (Genetic and molecular dissection of quantitative traits in rice, QT1001) and the Genebank Project of NIAS.

\section{Literature Cited}

Akagi,H., Y.Yokozeki, A.Inagaki and T.Fujimura (1997) Highly polymorphic microsatellites of rice consist of AT repeats, and a classification of closely related varieties with these microsatellite loci. Theor. Appl. Genet. 94: 61-67.

Chen,X., S.Temnykh, Y.Xu, Y.G.Cho, S.R.McCouch (1997) Development of a microsatellite framework map providing genomewide coverage in rice (Oryza sativa L.) Theor. Appl. Genet. 95: 553-567.

Charlesworth,B., P.Sniegowski and W.Stephean (1994) Evolutionary dynamics of repetitive DNA in Eukaryote. Science 371: 215220.

Cui,H., B.Peng, Z.Xing, G.Xu, B.Yu and Q.Zhang (2002) Molecular dissection of seedling-vigor and associated physiological traits in rice. Theor. Appl. Genet.105: 745-753.

Ebana,K., W.Yan, R.H.Dilday, H.Namai and K.Okuno (2001) Analysis of QTL associated with the allelopathic effect of rice using water-soluble extracts. Breed. Sci. 51: 47-51.

Frankel,O.H. (1984) Genetic perspectives of germplasm conservation. In: Arber,W.K., K.Llimensee, W.J.Peacock and P.Stralinger (eds.) Genetic Manipulation: Impact on Man and Society, Cambridge University Press, Cambridge, pp. 161-170.

Frankel,O.H. and M.E. Soule (1981) Conservation and Evolution. Cambridge Univ. Press. New York.

Fukuoka,S., D.S.Tran, K.Ebana, T.N.Luu, T.Nagamine and K.Okuno (2006) Genetic organization of aromatic rice as revealed by RAPD markers: A case study in conserving crop genetic resources on farm. Euphytica 149: 61-71.

Harushima,Y., M.Nakagahra, M.Yano, T.Sasaki and N.Kurata (2002) Diverse variation of reproductive barriers in three intraspecific rice crosses. Genetics 160: 313-322.

Ishimaru, K., M.Yano, N.Aoki, K.Ono, T.Hirose, S.Y.Lin, L.Monna, T.Sasaki and R.Ohsugi (2001) Toward the mapping of physiological and agronomic characters on a rice function map: QTL 
analysis and comparison between QTLs and expressed sequence tags. Theor. Appl. Genet. 102: 793-800.

Mu,P., C.Z.Li, C.P.Li., H.L.Zhang and X.K.Wang (2004) QTL analysis for lodging resistance in rice using a DH population under lowland and upland ecosystems. Acta genetica Sinica 31: 717723.

Murray,M.G. and W.F.Thompson (1980) Rapid isolation of high molecular weight plant DNA. Nuc. Acids Res. 8: 4321-4325.

Kobayashi,A., K.Ebana, S.Fukuoka and T.Nagamine (2006) Microsatellite markers revealed the genetic diversity of an old Japanese rice landrace 'Echizen'. Genet. Res. Crop Evol. 53:499-506.

Kojima,Y., K.Ebana, S.Fukuoka, T.Nagamine and M.Kawase (2005) Development of an RFLP-based rice diversity research set of germplasm. Breed. Sci. 55: 431-440.

Olsen, K., A.Caicedo, N. Polato, A. McClung, S. McCouch and M.Purugganan (2006) Selection under domestication: evidence for a sweep in the rice waxy genomic region. Genetics 173: 975-983.

Sato,T., T.Ueda, Y.Fukuta, T.Kumagai and M.Yano (2003) Mapping of quantitative trait loci associated with ultraviolet-B resistance in rice (Oryza sativa L.). Theor. Appl. Genet. 107: 1003-1008.

Sneath,P.H. and R.M.Sokal (1973) Numerical taxonomy. The principles and practice of numerical classification. Freeman and $\mathrm{Co}$, San Francisco, $359 \mathrm{p}$.

Sokal,R.R. and C. D.Michener (1958) A statistical method for evaluating systematic relationships. Univ. Kansas. Bull. 38: 14091438.

Tabien,E., Z.Li, H.Paterson, A.Marchetti, W.Stansel and M.Pinson
(2002) Mapping QTLs for field resistance to the rice blast pathogen and evaluating their individual and combined utility in improved varieties. Theor. Appl. Genet. 105: 313-324.

Tenmykh,S., W.Park, N.Ayres, S.Cattinhour, N.Hauch, L.Lipovich, Y.Cho, T.Ishii and S.McCouch (2000) Mapping and genome organization of microsatellite sequences in rice (Oryza sativa L.). Theor. Appl. Genet. 100: 697-712.

Temnykh,S., G.DeClerck, A.Lukashova, L.Lipovich, S.Cartinhour and S.McCouch (2001) Computational and experimental analysis of microsatellites in rice (Oryza sativa L.): frequency, length variation, transposon associations, and genetic marker potential. Genome Res. 11: 1441-1452.

Upadhyaya,H.D. and R.Ortiz (2001) A mini core subset for capturing diversity and promoting utilization of chickpea genetic resources in crop improvement. Theor. Appl. Genet. 102: 1292 1298.

Wissuwa, M., M. Yano and N.Ae (1998) Mapping of QTLs for phosphorus-deficiency tolerance in rice (Oryza sativa L.). Theor. Appl. Genet. 97: 777-783.

Yang, G.P., M.A.Saghai Maroof, C.G.Xu, Q.Zhang and R.M.Biyashev (1994) Comparative analysis of microsatellite DNA polymorphism in landraces and varieties of rice. Mol. Gen. Genet: 245: 187-194.

Zhang, G., T.S.Bharaj, Y.Lu, S.S.Virmani and N.Huang (1997) Mapping of the Rf-3 nuclear fertility-restoring gene for 'WA' cytoplasmic male sterility in rice using RAPD and RFLP markers. Theor. Appl. Genet. 94: 27-33. 